\title{
Transcriptional repression of Type I procollagen genes during adipocyte differentiation
}

\author{
TacGhee Yi ${ }^{1}$ Hee-Man Choi ${ }^{1}$, Rang-Woon Park ${ }^{1,2}$, \\ Kun-Young Sohn ${ }^{1}$ and In-San Kim ${ }^{1,2,3}$ \\ ${ }^{1}$ Department of Biochemistry, School of Medicine, Kyungpook National
University, 101 Dongin-dong, Jung-gu, Daegu 700-422, Korea
${ }^{2}$ National Research Laboratory, Kyungpook National University, 101
Dongin-dong, Jung-gu, Daegu 700-422, Korea
${ }^{3}$ Corresponding author: Tel, +82-53-420-6933;
Fax, +82-53-422-1466; E-mail, iskim@ @nu.ac.kr
}

Accepted 4 December 2001

Abbreviations: ECM, extracellular matrix; DEX, dexamethasone; IBMX, isobutylmethylxanthine; ORO, oil red $\mathrm{O}$

\begin{abstract}
Adipocyte differentiation is a very complex process in which whole-cell changes are accompanied. Among them, type I procollagen gene has been shown to specifically decrease during adipocyte differentiation; however, little is known about the molecular mechanism. To examine how type I procollagen gene expression is regulated at the level of transcription during adipocyte differentiation, 3T3-L1 preadipocyte cell line was used as an in vitro model. Northern blot analysis demonstrated that mRNA expression of type I procollagen gene was dramatically reduced during adipocyte differentiation. Time-course analysis indicated that decrease in mRNA expression occurred at early stage of differentiation. Studies on several stable cell lines showed that transcriptional activities of both $\alpha 1$ and $\alpha 2$ promoters decreased significantly during adipocyte differentiation. Despite extensive deletion-promoter analyses, however, we could not identify the cis-element responsible for the switch-off of type I procollagen gene during adipocyte differentiation, suggesting that the transcriptional repression of this gene occur through general transcription machinery rather than a specific cis-element. In conclusion, down-regulation of type I procollagen mRNA expression during adipocyte differentiation is due to repression of its promoter activity through general transcription machinery.
\end{abstract}

Keywords: Procollagen; adipocyte differentiation; transcription; repression

\section{Introduction}

Adipocyte differentiation is a very complex process that is triggered and promoted by coordinated signals of growth factors, cytokines, and hormones. Besides significant increases in mRNAs of the genes directly involved in lipidogenesis, changes occur in expression of cytoskeletal, extracellular matrix (ECM), and related components such as actin, tubulin, fibronectin, and collagen (Spiegelman and Farmer, 1982; Antras et al., 1989; Weiner et al., 1989). Modulation of cytoskeletal and ECM components could allow for cytoskeletal rearrangement, release of cell adhesion, and remodeling of cell components necessary for morphological changes. However, less attention has focused on the modulations in cytoskeletal and ECM components accompanying adipocyte differentiation. During differentiation, expressions of actin, tubulin, fibronectin, type I and type III collagen, and pref-1 decrease whereas expressions of CSPG-1, entactin/nidogen, and type IV collagen increase (Spiegelman and Farmer, 1982; Aratani and Kitagawa, 1988; Antras et al., 1989; Weiner et al., 1989; Calvo et al., 1991; Smas and Sul, 1993). Among these, collagen is the major ECM protein and shown to drastically decrease in mRNA level by $80-90 \%$ during 3T3-L1 differentiation (Weiner et al., 1989). However, little is known about the molecular mechanism of down-regulation of the collagen gene during adipocyte differentiation. Alteration in transcription of a gene implies that regulatory modulation may occur at the level of its promoter. We studied how type I procollagen gene transcription could be regulated at the level of its promoter during adipocyte differentiation, employing 3T3-L1 mouse preadipocyte cell line as an in vitro model system.

\section{Materials and Methods}

\section{Cell culture and differentiation}

3T3-L1 preadipocytes (ATCC CL-173 CCL-92.1) were maintained in Dulbecco's modified Eagles medium (DMEM) supplemented with $10 \%$ calf serum in a $37^{\circ} \mathrm{C}$ humidified incubator. Subconfluent cells were induced to differentiate to adipocytes by feeding with DMEM containing $10 \%$ fetal bovine serum (FBS) plus $1 \mu \mathrm{g} / \mathrm{ml}$ of insulin (Sigma, St. Louis, MO), $0.5 \mathrm{mM}$ of isobutylmethylxanthine (IBMX) (Sigma), and $0.25 \mu \mathrm{M}$ of dexamethasone (DEX) (Sigma). At $48 \mathrm{~h}$ postinduction, the cells were re-fed with the fresh medium containing FBS plus $1 \mu \mathrm{g} / \mathrm{ml}$ of insulin only. The cells were cultured for at 
least additional 3 days to further differentiate to mature adipocytes.

\section{Oil-Red O (ORO) staining}

Lipid accumulation in 3T3-L1 cells was identified by ORO staining. After 3T3-L1 preadipocytes were induced to differentiate into mature adipocytes, they were rinsed with phosphate-buffered saline (PBS), fixed with $70 \%$ ethanol for $5 \mathrm{~min}$., and then stained with ORO for $3 \mathrm{~min}$. Stained cells were extensively rinsed with PBS and distilled water. The cells were counter-stained with Harrishematoxylin for $1 \mathrm{~min}$ and then rinsed with distilled water.

\section{Northern blot hybridization}

Northern blot hybridization was done as described previously (Rhew et al., 1999). The cDNA probe used for hybridization was HF677, human $\alpha 1$ (I)procollagen cDNA (Chu et al., 1992).

\section{Plasmids}

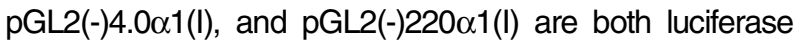
reporter plasmids containing $\alpha 1$ chain promoter constructs of mouse type I procollagen gene. To create pGL2 (-)4.0 1 (I), a $4.2 \mathrm{~kb}$-fragment cut from pJ400 (kindly provided by Dr. Benoit de Crombrugghe, MD Anderson Cancer Center, University of Texas, Houston, TX) by $\mathrm{BamHI}$ was inserted into $\mathrm{Bgl}$ II site of pGL2-Basic (Promega, Madison, WI). A fragment of 336 bp generated by Hind III-BgI II digestion from pG70 was subcloned to Hind III-Bgl II site of pGL2-Basic to produce pGL2(-) $220 \alpha 1(\mathrm{I})$. The other reporters containing several collagen promoter constructs were kindly provided by Dr. Benoit de Crombrugghe.

\section{Stable transfection and luciferase assay}

All transfections were performed by using LipofectAMINETM reagent (Life Technologies, Gaithersberg, MD) according to manufacturer's instructions. Cells were cotransfected with several luciferase reporter genes and a neomycinresistant selection marker gene. At $48 \mathrm{~h}$ posttransfection, the cells were subjected to selection with the medium containing $700 \mu \mathrm{g} / \mathrm{ml}$ of $\mathrm{G} 418$. The cells were re-fed with selection medium every three days until the colonies were apparently formed. At least more than 25 colonies were obtained from each transfection. The colonies were then pooled and cultured in the selection medium. Luciferase assay was carried out as described previously (Yi et al., 2000).

\section{Results}

As an in vitro model system for adipocyte differentiation study, a mouse preadipocyte cell line 3T3-L1 was employed. 3T3-L1 cells are easily induced to differentiate to adipocytes by combinatorial mixture of insulin, DEX, and
A

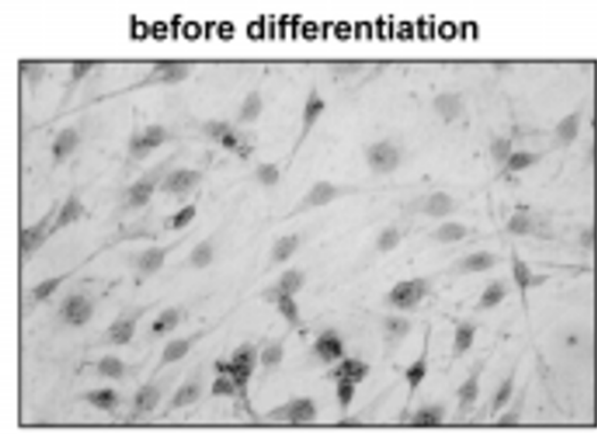

after differentiation

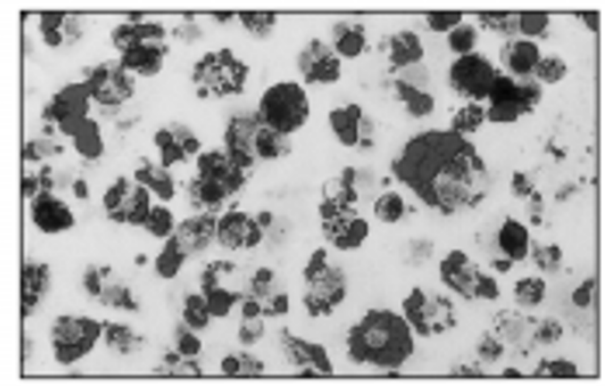

B

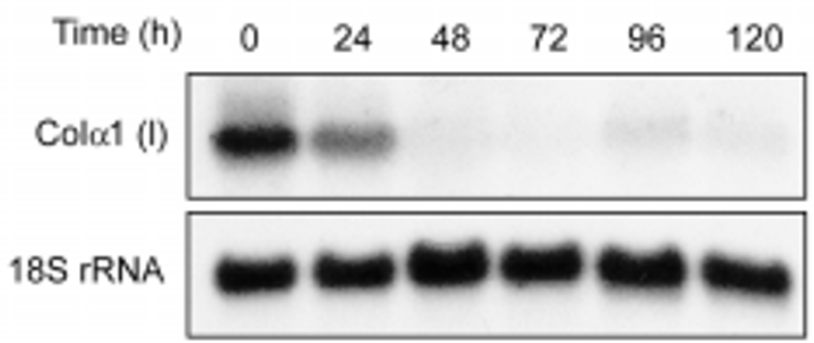

Figure 1. (A) Morphology of 3T3-L1 cells. left panel. 3T3-L1 preadipocytes. right panel. 3T3-L1 cells after induction to mature adipocytes. Lipid accumulation was visualized by ORO staining. ORO staining showed that no obvious lipid droplet was observed in preadipocytes whereas differentiated cells exhibited round shape characteristic to adipocytes and nearly all of them contained well-developing red-colored lipid droplets (magnitude: $\times 10$ ). (B) Decrease in mRNA in differentiated 3T3-L1 Cells. Northern hybridization was performed to examine whether mRNA expression is altered during adipocyte differentiation. After induction, 3T3-L1 cells were harvested at the indicated time. Northern blot analysis showed that MRNA level of differentiated 3T3-L1 cells is drastically decreased and that this occurs at early stage of differentiation. Col 1 1(I): $\alpha 1$ chain of type I procollagen. 
IBMX. To examine whether 3T3-L1 preadipocytes can be induced to fully differentiate to mature adipocytes in our system, adipocytes after induction were identified with ORO staining which selectively stains lipid-droplets. ORO staining showed that adipocyte differentiation of 3T3-L1 cells was well induced in our system (Figure 1A). Next, it was examined whether mRNA level of type I procollagen would be changed during adipocyte differentiation. Northern blot analysis showed that mRNA level of type I procollagen decreased as early as $24 \mathrm{~h}$ after induction and little signal was detected after $48 \mathrm{~h}$ (Figure 1B), suggesting that transcription of type I procollagen is down-regulated in the early stage of adipocyte differentiation. Our result of decreased transcription of type I procollagen gene during adipocyte differentiation is well consistent with the previous report (Weiner et al., 1989).

To test whether decreased mRNA level of type I collagen is due to decrease in its promoter activity, reporter plasmids containing type I procollagen promoter constructs were made (Figure 2A). First, 3T3-L1 preadipocytes were transfected with $\alpha 1$ chain promoter, pGL2(-)4.0 $\alpha 1(\mathrm{I})$, and $\alpha 2$ chain promoter, $\mathrm{pH} 5$, along with $\mathrm{pH} 2 \mathrm{Rneo}, \mathrm{a}$ neomycin-resistant gene-containing vector. Stable transfectants were selected with G418-containing selection medium for several weeks. At least more than 25 colonies were obtained and they were pooled. These stable transfectants were designated as L1pGL2(-)4.0 1 1(I) and L1pH5, respectively. After induced to mature adipocytes for 5 days, they were harvested and then assayed. Luciferase assay showed that the promoter activities of both cell lines were drastically reduced after differentiation (Figure 2B). Because mRNA level of type I procollagen decreased at early stage of differentiation (Figure 1B), we investigated whether its promoter activity is also suppressed at early stage of differentiation to cause decrease in mRNA expression. L1pH5 was induced to differentiate, harvested at the indicated times, and then assayed. Upon differentiation, the promoter activity decreased even at $24 \mathrm{~h}$ (Figure 2C). Consistent with the Northern

A
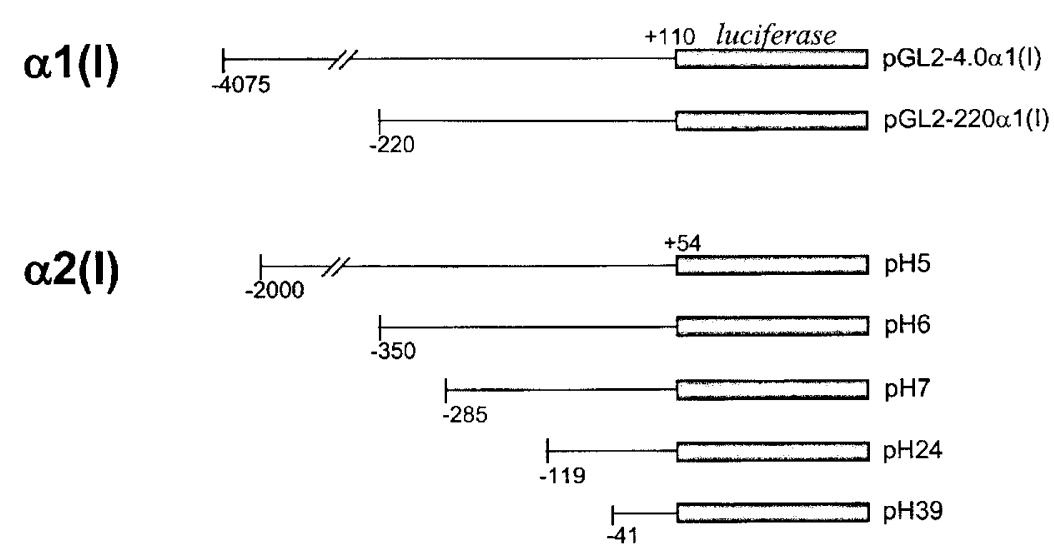

B

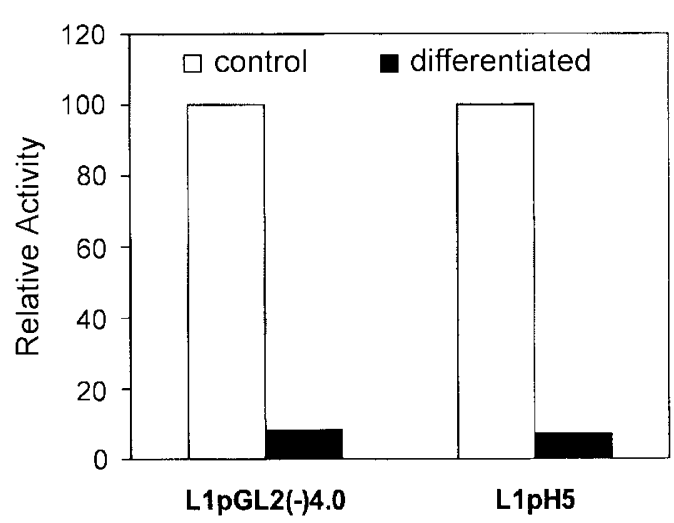

C

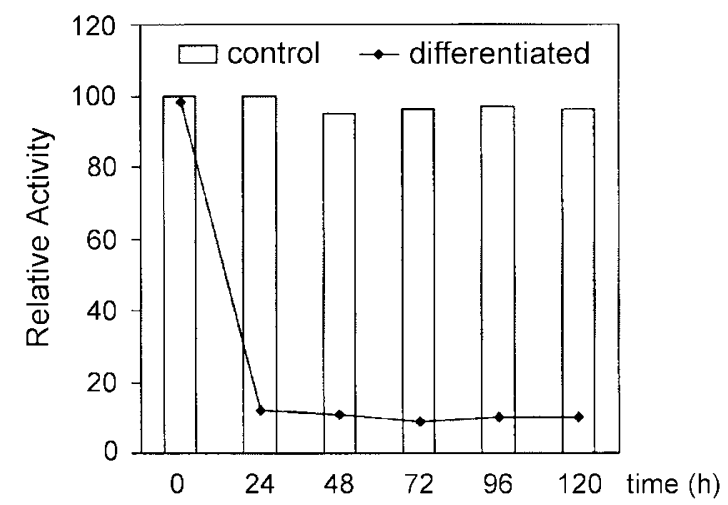

Figure 2. (A) Luciferase reporter genes containing several deletion promoters of type I procollagen. (B) Transrepression of type I procollagen promoters during adipocyte differentiation. At 5 days postinduction, the indicated stable cell lines expressing pGL2(-)4.0 or pH5 were harvested and assayed for promoter acitivities. When differentiated, the promoter activities of both cell lines were drastically reduced. (C) The promoter activity of L1pH5 was significantly reduced within $24 \mathrm{~h}$ after induction, which is well consistent with the Northern blot analysis. 
blot analysis, these results demonstrated that early down-regulation of type I procollagen mRNA expression is due to its decreased promoter activity at early stage of adipocyte differentiation.

We hypothesized that if the cis-element responsible for the switch-off of type I procollagen gene transcription during adipocyte differentiation may reside in its promoter, cis-element-truncated mutant promoter would not respond to differentiation signals. To ascertain our hypothesis, a series of sequential deletion mutant promoter constructs was made (Figure 2A). 3T3-L1 preadipocytes were transfected with each of promoter constructs along with $\mathrm{pH} 2 \mathrm{Rneo}$. Stable transfectants were made and selected. L1pGL2(-)220 $\alpha 1$ (I), and L1pGL2(-)30 were 3T3-L1-derived cell lines stably transfected with type I collagen $\alpha 1$ chain promoter constructs of pGL2(-)220 $\alpha 1(\mathrm{I})$, and pGL2(-) $30 \alpha 1(\mathrm{I})$, respectively. L1pH6, L1pH7, L1pH24, and L1pH39 were 3T3-L1-derived cell lines stably transfected with type I collagen $\alpha 2$ chain promoter constructs of $\mathrm{pH} 6$, $\mathrm{pH} 7, \mathrm{pH} 24$, and $\mathrm{pH} 39$, respectively. Each of these cell lines along with L1pGL2(-)4.0 1 (I) and L1pH5 was induced to differentiate to adipocytes. At 5 days post induction, the cells were harvested and assayed for luciferase activity. When differentiated, the promoter activity of pGL2(-)220 1 1(I) was decreased as much as that of pGL2(-)4.0 1 1(I) (Figure 3A). Decrease in the promoter activity was also observed in $\alpha 2$ chain reporters-transfected cell lines. The promoter activities of all of constructs from $\mathrm{pH} 5$ to $\mathrm{pH} 24$ were also reduced significantly (Figure 3B). The activity reduction of $\mathrm{L} 1 \mathrm{pH} 24$ was not so much as those of the others. Maybe this is thought to be caused by its lower extent of differentiation, not by de-repression. Result from the shortest pGL2(-)30 1 1(I) and $\mathrm{pH} 39$ containing just the minimal promoter was not shown because their basal activities were nearly the background. Taken together, these data suggest that the cis-element may be present within $220 \mathrm{bp}$ of $\alpha 1$ promoter and within $119 \mathrm{bp}$ of $\alpha 2$

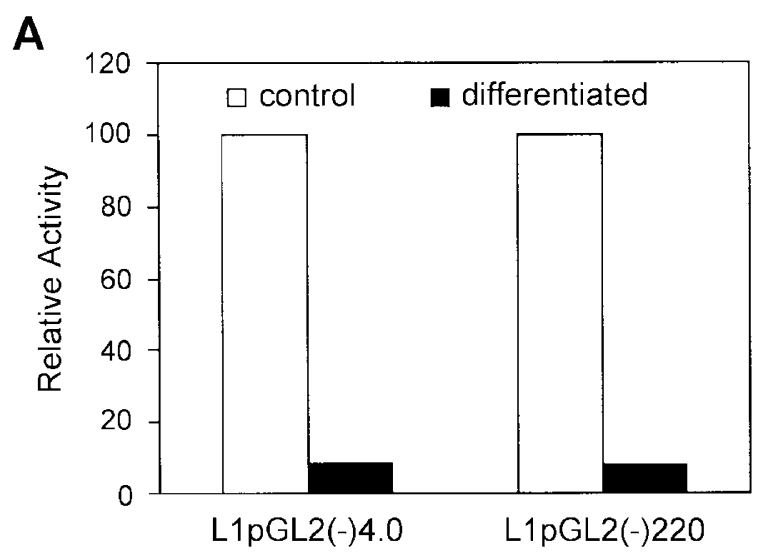

promoter of type I procollagen gene.

To examine whether cis-element may be present within 119 bp of $\alpha 2$ promoter, we made additional stable cell lines overexpressing internal deletion promoter reporters. pTH13 and pTH5 are luciferase reporters of promoters in which the segment between 130 and $40 \mathrm{bp}$ is internally deleted from $\mathrm{pH} 5$ and $\mathrm{pH} 6$, respectively (Figure 4A). L1pTH13 and L1pTH5 are 3T3-L1-derived cell lines stably expressing pTH5 and pTH6, respectively. After induced to differentiate to adipocytes, the cells were harvested and assayed for the promoter activity. The promoter activities of differentiated cells were still reduced so much as those of their parental constructs (Figure 4B), suggesting that during adipocyte differentiation, type I procollagen gene may be regulated by a mechanism governing differentiation-dependent transcriptional repression other than a regulatory mechanism exerted by a master cis-element.

\section{Discussion}

Preadipocyte conversion to fat-rich adipocyte is a very complicated process requiring whole-cell alterations in cellular metabolism, morphology, gene expression, and so on. Although more than 300 proteins were shown to be dramatically altered during 3T3-L1 differentiation, the majority of these have not been characterized yet (Sadowski et al., 1992). The molecular mechanisms governing transcriptional regulation during adipocyte differentiation have been addressed for only a few of these genes. Studies to date have largely focused on transcriptional activation during adipocyte differentiation. More intention has been given to lipidogenesis or lipid metabolism-related cellular changes whereas little is known about the regulation of ECM or cytoskeletal genes of which modulations account for substantial changes in architectural and structural complexity of

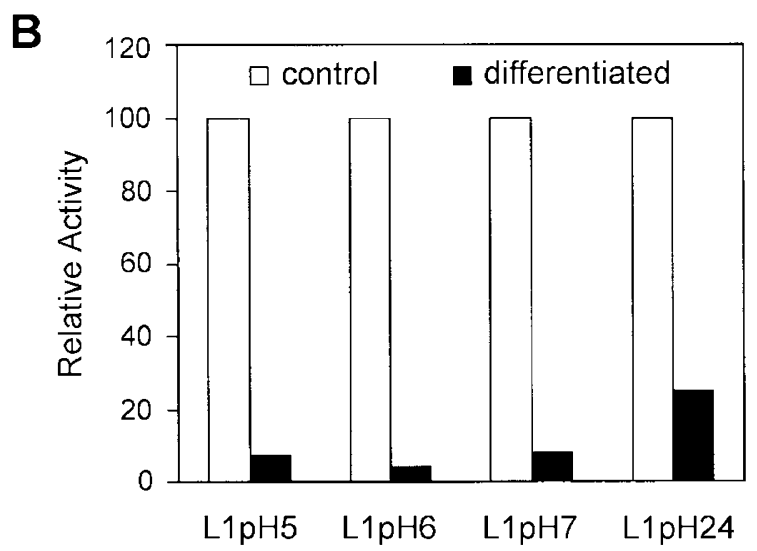

Figure 3. Sequential deletion-promoter analysis of type I procollagen promoters during adipocyte differentiation. (A) Col $\alpha 1(\mathrm{I})$. (B) Col $\alpha 2(\mathrm{I})$. Each of stable transfectants was induced to differentiate to adipocyte. At 5 days postinduction, the cells were harvested and assayed. When differentiated, the promoter activities of all cell lines were still significantly reduced. 
A

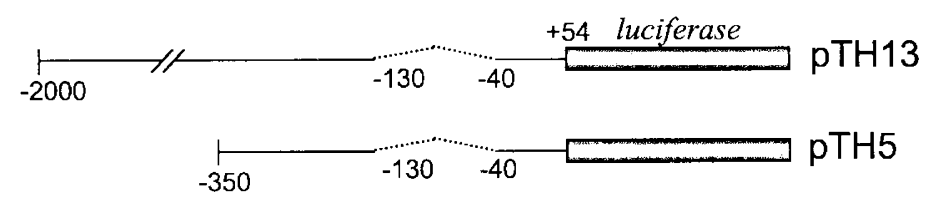

B

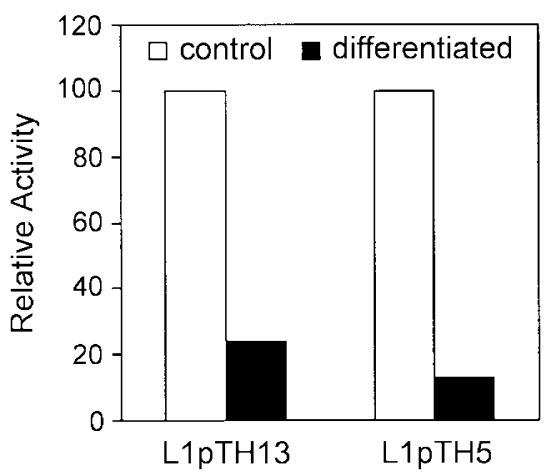

Figure 4. Analysis of internal deletion mutant promoters of Cola2(I) during adipocyte differentiation. (A) Schematic structures of internal deletion promoter constructs of pTH13 and pTH5. (B) Both of stable transfectants was induced to differentiate to adipocyte. At 5 days postinduction, the cells were harvested and assayed. The promoter activities of p TH13 and pTH5 was still observed to decrease so much as their parental constructs, pH5 and pH6, respectively.

adipocytes (Chapman et al., 1984; Bernlohr et al., 1985; Cook et al., 1985). Moreover, genes that are repressed during adipocyte differentiation have been given less attention whereas transcriptional activation of genes has received extensive attention. ECM is not merely a structural or an architectural entity, but plays an important role in transducing signals into cells for various cell behaviors. Collagen is the most abundant component of ECM and serves as substratum for cell adhesion, migration, spreading, and anchorage-dependent growth (Culp et al., 1979; Yamada, 1982; Spiegelman and Ginty, 1983). Prior to differentiation, 3T3-L1 preadipocytes resemble typical fibrobalsts. Once induced to differentiate, cells become thinner and enlarged followed by assuming signet-ring appearance of mature adipocytes. These morphological changes imply alterations in ECM protein expression. Actually, type I procollagen gene was shown to decrease in its mRNA level during adipocyte differentiation, and that was also confirmed in this study. Our study demonstrated that down-regulation of type I procollagen mRNA expression is caused by its decreased promoter activity during adipocyte differentiation. Transcriptional repression of type I procollagen is specific to preadipocyte cells; there was no repression observed in a non-adipogenic fibroblast cell line C3H10T1/2 which was stably transfeced with $\mathrm{pH} 5$ when treated with the differentiation-inducing reagents (data not shown). We tried to identify the cis-element that governs gene transcription of type I procollagen during adipocyte differentiation. Deletion promoter analysis, however, showed that there is no cis-element responsible for the switch on/off of type I procollagen gene.

Repression mechanisms are less understood than activation mechanisms. Generally, transcriptional repression can be categorized as the followings (Herschbach and Johnson, 1993; Johnson, 1995; Hanna-Rose and Hansen, 1996); first, inactivation of an activator causes repression through posttranslational modification of the activator, dimerization of the activator with a nonfunctional partner, competition for the activator's binding site, or a direct interaction between repressor and activator (Benezra et al., 1990; Momand et al., 1992; Munshi et al., 1998). Second, repression can be accomplished by repressor proteins that associate with general transcription factors and thereby inhibit the formation of pre-initiation complex (Orphanides, 1996). Third, repression can be mediated by a specific DNA element and sequence-specific DNAbinding repressor protein (Hanna-Rose and Hansen, 1996). No cis-element and nuclear proteins responsible for repression of preadipocyte genes during adipocyte differentiation have been identified except for pref-1 gene (Smas et al., 1998). In the case of type I procollagen, it is deduced from our data that transcriptional repression may not be mediated by a certain DNA element of the promoter. Rather, repression may occur through general transcription machinery directly modulated by differentiation signals in this gene. Also it is possible that differentiation signals may down-regulate expression of transcription factors responsible for the basal expression of type I procollagen, thereby switching-off the collagen gene transcription. However, these possibilities remain to be investigated.

Several classes of transcription factors have been shown to be important in adipocyte conversion (MacDougald and Lane, 1995; Brun et al., 1996; Fajas et al., 1998). Of these, PPAR $\gamma$ and $\mathrm{C} / \mathrm{EBP} \alpha$ have been demonstrated as key regulators both in adipocyte differentiation and in maintaining adipocyte phenotype. PPAR $\gamma$ is strongly induced during adipocyte differentiation and activates several adipocyte-specific genes (Spiegelman and Flier, 1996; Mandrup and Lane, 1997). C/EBP $\alpha$ binds to and transactivates the promoters of many genes that are specifically expressed during adipocyte differentiation (Christy et al., 1989; Kaestner et al., 1990). Although these findings revealed that PPAR and C/EBP families are crucial for adipocyte differentiation, it is unlikely that they affect transcriptional repression of type I procollagen gene. Time-course showed that 
significant decrease in type I procollagen mRNA was observed within $24 \mathrm{~h}$ whereas it has been demonstrated that PPAR $\gamma$ and $\mathrm{C} / \mathrm{EBP} \alpha$ are induced at later stage and their expressions are maximal at terminal stage (MacDougald and Lane, 1995; Cowherd et al., 1999). Actually, transient transfection showed that overexpression of PPAR $\gamma$ and its heterodimer partner $\mathrm{RXR} \alpha$ did not affect the promoter activity of type I procollagen gene (data not shown). Therefore, it would be speculated that transcriptional repression of type I procollagen gene is requisite for adipocyte differentiation and not governed by these master regulatory proteins that promotes adipocyte differentiation.

Taken together, this work demonstrates that downregulation of type I procollagen gene during adipocyte differentiation is apparently caused by repression of its promoter activity and suggests that transcriptional repression may be mediated by general transcription machinery rather than a specific-DNA element on the promoter.

\section{Acknowledgement}

We thank Dr. Benoit de Crombrugghe (MD Anderson Cancer Center, University of Texas, Houston, TX) for the collagen promoter constructs. This work was supported by Medical Research Institute of Kyungpook National University Hospital and Hankok Medical Science Foundation.

\section{References}

Antras J, Hilliou F, Redziniak G, Pairault J. Decreased biosynthesis of actin and cellular fibronectin during adipose conversion of 3T3-F442A cells: reorganization of the cytoarchitecture and extracellular matrix fibronectin. Biol Cell 1989;66:247-54

Aratani, Y, Kitagawa, Y. Enhanced synthesis and secretion of type IV collagen and entactin during adipose conversion of 3T3-L1 cells and production of unorthodox laminin complex. $J$ Biol Chem 1988;263:16163-69

Benezra R, Davis RL, Lockshon D, Turner DL, Weintraub H. The protein Id: a negative regulator of helix-loop-helix DNA binding proteins. Cell 1990;61:49-59

Bernlohr DA, Bolanowski MA, Kelly TJ, Jr, Lane MD. Evidence for an increase in transcription of specific mRNAs during differentiation of 3T3-L1 preadipocytes. J Biol Chem 1985;260: 5563-67

Brun RP, Kim JB, Hu E, Altiok S, Spiegelman BM. Adipocyte differentiation: a transcriptional regulatory cascade. Curr Opin Cell Biol 1996;8:826-32

Calvo JC, Rodbard D, Katki A, Chernick S, Yanagishita M. Differentiation of 3T3-L1 preadipocytes with 3-isobutyl-1methylxanthine and dexamethasone stimulates cell-associated and soluble chondroitin 4-sulfate proteoglycans. J Biol Chem 1991;266:11237-45

Chapman AB, Knight DM, Diekmann BS, Ringold GM. Analysis of gene expression during differentiation of adipogenic cells in culture and hormonal control of the developmental program. J Biol Chem 1984;259:15548-55

Christy RJ, Yang VW, Ntambi JM, Geiman DE, Landschulz WH, Friedman AD, Nakabeppu Y, Kelly TJ, Lane MD. Differentiation-induced gene expression in 3T3-L1 preadipocytes: CCAAT/enhancer binding protein interacts with and activates the promoters of two adipocyte-specific genes. Genes Dev 1989;3:1323-35

Chu ML, Myers JC, Bernard MP, Ding JF, Ramirez F. Cloning and characterization of five overlapping CDNAs specific for the human pro $\alpha 1$ (I) collagen chain. Nucleic Acid Res. 1992;10: 5925-34

Cook KS, Hunt CR, Spiegelman BM. Developmentally regulated mRNAs in 3T3-adipocytes: analysis of transcriptional control. J Cell Biol 1985;100:514-20

Cowherd RM, Lyle RE, McGehee REJr. Molecular regulation of adipocyte differentiation. Semin Cell Dev Biol 1999;10:3-10

Culp LA, Murray BA, Rollins BJ Fibronectin and proteoglycans as determinants of cell-substratum adhesion. J Supramol Struct 1979;11:401-27

Fajas L, Fruchart J-C, and Auwerx J. Transcriptional control of adipogenesis. Curr Opin Cell Biol 1998;10:165-73

Hanna-Rose W, Hansen U. Active repression mechanisms of eucaryotic transcription repressior. Trends Genet 1996;12: 229-34

Herschbach BM, Johnson AD. Transcriptional repression in eucaryotes. Annu Rev Cell Biol 1993;9:479-509

Johnson AD. The price of repression. Cell 1995;81:655-58

Kaestner KH, Christry RJ, Lane, MD. Mouse insulin-responsive glucose transporter gene: characterization of the gene and trans-activation by the CCAAT/enhancer binding protein. Proc Natl Acad Sci USA 1990;87:251-55

MacDougald OM, Lane MD. Transcriptional regulation of gene expression during adipocyte differentiation. Annu Rev Biochem 1995;64:345-73

Mandrup S, Lane MD. Regulating adipogenesis. J Biol Chem 1997;272:5367-70

Momand J, Zambetti GP, Olson DC, George D, Levine AJ. The mdm-2 oncogene product forms a complex with the p53 protein and inhibits p53-mediated transactivation. Cell 1992; 69:1237-45

Munshi N, Merika M, Yie J, Senger K, Chen G, Thanos D. Acetylation of $\mathrm{HMGI}(\mathrm{Y})$ by CBP turns off IFN $\beta$ expression by disrupting enhanceosome. Mol Cell 1998;2:457-67

Orphanides $G$, Lagrange T, Reinberg $D$. The general transcription factors of RNA polymerase II. Genes Dev 1996;10:2657-83

Rhew J-H, Shin Y-A, Lee B-H, Park R-W, Kim I-S. Induction of fibronectin gene expression by inhibitors of protein phosphatase type $2 \mathrm{~B}$ in normal and transformed fibroblasts. 
Exp. Mol. Med. 1999;31:71-75

Sadowski HB, Wheeler TT, Young DA. Gene expression during 3T3-L1 adipocyte differentiation: characterization of initial responses to the inducing agents and changes during commitment to differentiation. J Biol Chem 1992;267:4722-31

Smas CM, Kachinskas D, Liu C-M, Xie X, Dircks LK, Sul HS. Transcriptional control of the pref-1 gene in 3T3-L1 adipocyte differentiation: sequence requirement for differentiationdependent suppression. J Biol Chem 1998;273:31751-58

Smas CM, Sul HS. Pref-1, a protein containing EGF-like repeats, inhibits adipocyte differentiation. Cell 1993;73:725-34

Spiegelman BM, Farmer SR. Decreases in tubulin and actin gene expression prior to morphological differentiation of 3T3 adipocytes. Cell 1982;29:53-60
Spiegelman BM, Ginty CA. Fibronectin modulation of cell shape and lipogenic gene expression in 3T3-adipocytes. Cell 1983;35:657-66

Spiegelman BM, Flier JS. Adipogenesis and obesity: rounding out the big picture. Cell 1996;87:377-80

Weiner FR, Shah A, Smith PJ, Rubin CS. Regulation of collagen gene expression in 3T3-L1 cells:effects of adipocyte differentiation and tumor necrosis factor- $\alpha$. Biochemistry 1989;28:4094-99

Yamada KM. in Cell Biology of the Extracellular Matrix (Hay, E.D. Ed.) pp.95-114, Plenum press, NY, 1982

Yi T-G, Lee B-H, Park R-W, Kim I-S. Transactivation of fibronectin promoter by HTLV-1 Tax through NF-kB pathway. Biochem Biophys Res Commun 2000;276:579-86 